UCRLDetermination of the standard deviation on multiplication based on count distributions

Verbeke, Jerome

July 10, 2009 
This document was prepared as an account of work sponsored by an agency of the United States government. Neither the United States government nor Lawrence Livermore National Security, LLC, nor any of their employees makes any warranty, expressed or implied, or assumes any legal liability or responsibility for the accuracy, completeness, or usefulness of any information, apparatus, product, or process disclosed, or represents that its use would not infringe privately owned rights. Reference herein to any specific commercial product, process, or service by trade name, trademark, manufacturer, or otherwise does not necessarily constitute or imply its endorsement, recommendation, or favoring by the United States government or Lawrence Livermore National Security, LLC. The views and opinions of authors expressed herein do not necessarily state or reflect those of the United States government or Lawrence Livermore National Security, LLC, and shall not be used for advertising or product endorsement purposes.

This work performed under the auspices of the U.S. Department of Energy by Lawrence Livermore National Laboratory under Contract DE-AC52-07NA27344. 
LLNL-TR-414591

\title{
Determination of the standard deviation on multiplication based on count distributions
}

\author{
Jerome M. Verbeke* \\ Lawrence Livermore National Laboratory
}

June 1, 2009

\section{Contents}

1 Introduction $\quad 2$

2 Methodology 2

$\begin{array}{lll}3 & \text { Assumptions made for the analysis } & 7\end{array}$

4 Nuclear data $\quad 7$

5 Simulations $\quad 7$

6 Overlapping time gates $\quad 8$

6.1512 time gates in arithmetic progression from 1 to $512 \mu \mathrm{s} \ldots \ldots \ldots \ldots$

6.2512 time gates in arithmetic progression from 4 to $2000 \mu \mathrm{s} \ldots \ldots \ldots$

7 Non-overlapping time gates $\quad 10$

7.157 non-overlapping time gates in geometric progression from $1 \mathrm{~ns}$ to $524 \mu \mathrm{s} \ldots \ldots$

7.260 non-overlapping time gates in geometric progression from $1 \mathrm{~ns}$ to $1048 \mu \mathrm{s} \ldots \ldots$

7.363 non-overlapping time gates in geometric progression from $1 \mathrm{~ns}$ to $2096 \mu \mathrm{s} \ldots \ldots$

8 Conclusion $\quad 12$

$\begin{array}{ll}\text { References } & 14\end{array}$

${ }^{*}$ Contact info: verbeke2@1lnl.gov, 925-422-8337 


\section{Introduction}

The multiplication $\mathrm{M}$ of a multiplying object can be determined by analyzing the arrival times of thermal neutrons in detectors such as helium tubes. A source emitting neutrons randomly will lead to neutron inter arrival times obeying Poisson process statistics. By observing how the inter arrival times of detected counts differ from Poisson statistics, one can assess properties of the neutron source such as the source intensity and multiplication.

While the method of analyzing the inter arrival times of neutrons using count distributions to determine characteristics of the source has been used for a long time, the treatment of the errors on the determined quantities multiplication $\mathrm{M}$ and source strength $\mathrm{S}$ have not yet been adequately treated. This object of this report is to assess these errors. ${ }^{1}$

\section{Methodology}

To compute the multiplication $\mathrm{M}$ and the source strength $\mathrm{S}$ of an object, we first divide the data stream into $\mathrm{N}$ non overlapping segments of equal width $\mathrm{T}$. By counting the number of detected neutrons in each of the segments, we can build a count distribution. Normalizing this count distribution by the number of segments $\mathrm{N}$, we get $b_{n}(T)$, the probability of getting $n$ counts in a time gate $\mathrm{T}$. A typical count distribution is shown in Fig. 1 for $\mathrm{T}=512 \mu$ seconds.

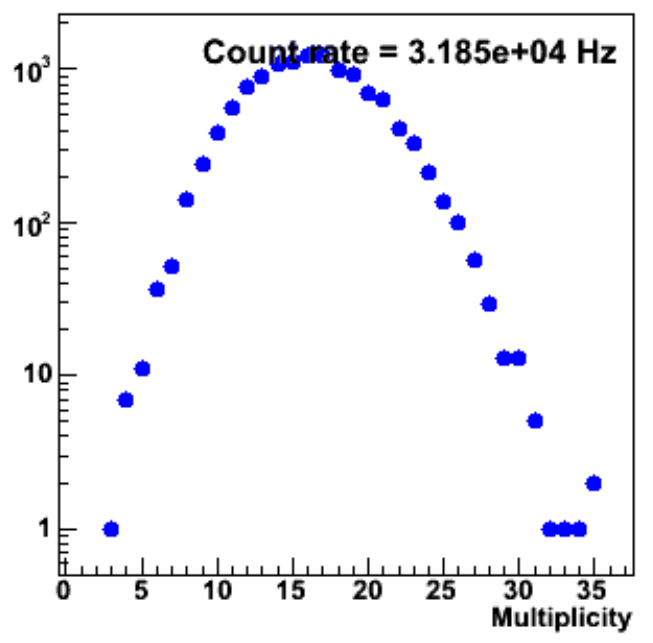

Figure 1: Example of a random time gate count distribution with $\mathrm{T}=512 \mu$ seconds

\footnotetext{
${ }^{1}$ This document was prepared as an account of work sponsored by an agency of the United States government. Neither the United States government nor Lawrence Livermore National Security, LLC, nor any of their employees makes any warranty, expressed or implied, or assumes any legal liability or responsibility for the accuracy, completeness, or usefulness of any information, apparatus, product, or process disclosed, or represents that its use would not infringe privately owned rights. Reference herein to any specific commercial product, process, or service by trade name, trademark, manufacturer, or otherwise does not necessarily constitute or imply its endorsement, recommendation, or favoring by the United States government or Lawrence Livermore National Security, LLC. The views and opinions of authors expressed herein do not necessarily state or reflect those of the United States government or Lawrence Livermore National Security, LLC, and shall not be used for advertising or product endorsement purposes.

This work performed under the auspices of the U.S. Department of Energy by Lawrence Livermore National Laboratory under Contract DE-AC52-07NA27344.
} 
We assume that each data point in the count distribution $b_{n}$ is a binomially distributed random variable, so that if we define

$$
C_{n}=N b_{n}+\varepsilon_{n}
$$

where $\varepsilon_{n}$ is the error on the data point, the variance on each point is

$$
\operatorname{var}\left(b_{n}\right)=\left\langle\varepsilon_{n}^{2}\right\rangle=N b_{n}\left(1-b_{n}\right)
$$

where $\angle$.$\rangle stands for taking the ensemble average over a number of experiments. Another quantity of$ importance is the covariance between the errors $\varepsilon_{n}$ on different $b_{n}$. Given the constraint that the $C_{n}$ are bound by

$$
\sum_{n=0}^{\infty} C_{n}(T)=N
$$

the sum of all the errors $\varepsilon_{n}$ is zero for any given experiment. Therefore, there exists a negative correlation between the errors $\varepsilon_{n}$, an increase in one component requires a decrease in another component. It can be shown (as in Ref. [1]) that the covariance between these errors is

$$
\left\langle\varepsilon_{n 1} \varepsilon_{n 2}\right\rangle=-N b_{n 1} b_{n 2}
$$

The first moment of the count distribution $b_{n}$ is the average number of events counted in a fixed time gate width T:

$$
\bar{C}(T)=\sum_{n=0}^{\infty} n b_{n}(T)
$$

The variance on this number can be calculated by propagating the errors $\varepsilon_{n}$ on the $b_{n}$ distribution via Eq. 5 :

$$
\begin{aligned}
C & =\frac{1}{N} \sum_{n=0}^{\infty} n C_{n} \\
& =\frac{1}{N} \sum_{n=0}^{\infty} n\left(N b_{n}+\varepsilon_{n}\right) \\
& =\bar{C}+\frac{1}{N} \sum_{n=0}^{\infty} n \varepsilon_{n}
\end{aligned}
$$

For the sake of clarity later in the text, we define

$$
\delta C=C-\bar{C}=\frac{1}{N} \sum_{n=1}^{\infty} n \varepsilon_{n}
$$

Squaring $\mathrm{C}$ in Eq. 7, we get

$$
C^{2}=\bar{C}^{2}+2 \frac{\bar{C}}{N} \sum_{n=0}^{\infty} n \varepsilon_{n}+\frac{1}{N^{2}}\left(\sum_{n=0}^{\infty} n^{2} \varepsilon_{n}^{2}+2 \sum_{n 1<n 2} n_{1} n_{2} \varepsilon_{n_{1}} \varepsilon_{n_{2}}\right)
$$


Taking the ensemble average over a number of experiments, the averages of the individual errors $\varepsilon_{n}$ are equal to zero $\left(\left\langle\varepsilon_{n}\right\rangle=0\right)$ and we thus have

$$
\left\langle C^{2}\right\rangle=\bar{C}^{2}+0+\frac{1}{N^{2}}\left(\sum_{n=0}^{\infty} n^{2} N b_{n}\left(1-b_{n}\right)-2 N \sum_{n 1<n 2} n_{1} n_{2} b_{n_{1}} b_{n_{2}}\right)
$$

where we have used Eqs. 2 and 4. Redistributing the terms, we finally get the variance on C:

$$
\begin{aligned}
\left\langle(C-\bar{C})^{2}\right\rangle & =\left\langle C^{2}\right\rangle-\bar{C}^{2} \\
& =\frac{1}{N}\left(\sum_{n=1}^{\infty} n^{2} b_{n}-\left(\sum_{n=0}^{\infty} n b_{n}\right)^{2}\right)
\end{aligned}
$$

$\bar{C}(T)$ is plotted as a function of the time gate width T as in Fig. 2. The variance on $\bar{C}$ is so small that the error bars can not be seen on the graph without zooming in.

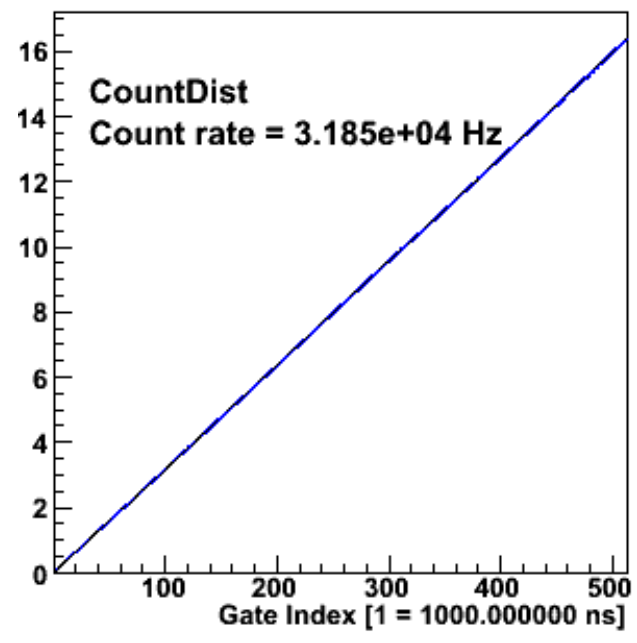

Figure 2: First moment of the random time gate count distribution as a function of the time gate width

Knowing that the first moment is also equal to

$$
\begin{aligned}
\bar{C}(T) & =\varepsilon\left(M-\frac{M-1}{\bar{v}}\right) S T \\
& =R_{1} T
\end{aligned}
$$

where $\mathrm{S}$ is the source strength in neutrons per second, the count rate $R_{1}$ can be extracted by fitting the points in Fig. 2 with the straight line given by Eq. 11.

The Feynman variance $\overline{Y_{2 F}}(T)$ of $b_{n}$ is defined as

$$
\overline{Y_{2 F}}(T)=\frac{\overline{Y_{2}}(T)}{\bar{C}(T)}
$$


where

$$
\overline{Y_{2}}(T)=\sum_{n=0}^{\infty}\left(\begin{array}{l}
n \\
2
\end{array}\right) b_{n}(T)-\sum_{n=0}^{\infty}\left(\begin{array}{l}
n \\
2
\end{array}\right) \mathbb{P}_{n}(T)
$$

and $\mathbb{P}_{n}(T)$ is the Poisson probability of getting $\mathrm{n}$ counts within time gate $\mathrm{T}$ for the count rate determined above. The second combinatorial moment of the Poisson probability will be derived first $\left(\mathbb{P}_{n}(T)\right.$ will be replaced by $\mathbb{P}_{n}$ for the sake of simplicity):

$$
\begin{aligned}
\sum_{n=2}^{\infty}\left(\begin{array}{c}
n \\
2
\end{array}\right) \mathbb{P}_{n} & =\sum_{n=2}^{\infty}\left(\begin{array}{c}
n \\
2
\end{array}\right) \frac{C^{n}}{n !} e^{-C} \\
& =\frac{1}{2} \sum_{n=2}^{\infty} \frac{C^{n}}{(n-2) !} e^{-C} \\
& =\frac{C^{2}}{2} \sum_{m=0}^{\infty} \frac{C^{m}}{(m) !} e^{-C} \\
& =\frac{C^{2}}{2}
\end{aligned}
$$

The variances on $\overline{Y_{2}}(T)$ and $\overline{Y_{2 F}}(T)$ will be derived in turn:

$$
\begin{aligned}
Y_{2} & =\frac{1}{N} \sum_{n=2}^{\infty}\left(\begin{array}{l}
n \\
2
\end{array}\right) C_{n}-\sum_{n=2}^{\infty}\left(\begin{array}{c}
n \\
2
\end{array}\right) \mathbb{P}_{n} \\
& =\frac{1}{N} \sum_{n=2}^{\infty}\left(\begin{array}{l}
n \\
2
\end{array}\right) N b_{n}+\frac{1}{N} \sum_{n=2}^{\infty}\left(\begin{array}{l}
n \\
2
\end{array}\right) \varepsilon_{n}-\frac{C^{2}}{2} \\
& =\frac{1}{N} \sum_{n=2}^{\infty}\left(\begin{array}{l}
n \\
2
\end{array}\right) N b_{n}+\frac{1}{N} \sum_{n=2}^{\infty}\left(\begin{array}{l}
n \\
2
\end{array}\right) \varepsilon_{n}-\frac{1}{2}\left(\bar{C}+\frac{1}{N} \sum_{n=1}^{\infty} n \varepsilon_{n}\right)^{2} \\
& =\frac{1}{N} \sum_{n=2}^{\infty}\left(\begin{array}{l}
n \\
2
\end{array}\right) N b_{n}-\frac{\bar{C}^{2}}{2}+\frac{1}{N} \sum_{n=2}^{\infty}\left(\begin{array}{c}
n \\
2
\end{array}\right) \varepsilon_{n}-\frac{\bar{C}}{N} \sum_{n=1}^{\infty} n \varepsilon_{n}-\frac{1}{2 N^{2}}\left(\sum_{n=1}^{\infty} n \varepsilon_{n}\right)^{2}
\end{aligned}
$$

where the last term is of $2^{\text {nd }}$ order and can safely be ignored.

$$
Y_{2}=\overline{Y_{2}}+\frac{1}{N} \sum_{n=1}^{\infty}\left[\left(\begin{array}{l}
n \\
2
\end{array}\right)-\bar{C} n\right] \varepsilon_{n}
$$

For the sake of clarity, we define

$$
\delta Y_{2}=Y_{2}-\overline{Y_{2}}=\frac{1}{N} \sum_{n=1}^{\infty}\left[\left(\begin{array}{l}
n \\
2
\end{array}\right)-\bar{C} n\right] \varepsilon_{n}
$$


We now turn to calculating the variance on $Y_{2 F}(T)$

$$
\begin{aligned}
\left(Y_{2 F}-\overline{Y_{2 F}}\right)^{2} & =\left(\frac{Y_{2}}{C}-\frac{\overline{Y_{2}}}{\bar{C}}\right)^{2} \\
& =\left(\frac{\left(\overline{Y_{2}}+\delta Y_{2}\right) \bar{C}-\overline{Y_{2}}(\bar{C}+\delta C)}{C \bar{C}}\right)^{2} \\
& =\left(\frac{\delta Y_{2} \bar{C}-\overline{Y_{2}} \delta C}{C \bar{C}}\right)^{2} \\
& =\frac{\delta Y_{2}^{2} \bar{C}^{2}+{\overline{Y_{2}}}^{2} \delta C^{2}-2 \delta Y_{2} \overline{C Y_{2}} \delta C}{(C \bar{C})^{2}} \\
& =\frac{\delta Y_{2}^{2}}{C^{2}}+\frac{{\overline{Y_{2}}}^{2} \delta C^{2}}{C^{2} \bar{C}^{2}}-2 \frac{\delta Y_{2} \overline{Y_{2}} \delta C}{C \bar{C}}
\end{aligned}
$$

Neglecting higher order terms, we get

$$
\left(Y_{2 F}-\overline{Y_{2 F}}\right)^{2}={\overline{Y_{2 F}}}^{2}\left(\left(\frac{\delta Y_{2}}{\overline{Y_{2}}}\right)^{2}+\left(\frac{\delta C}{C}\right)^{2}-2 \frac{\delta Y_{2} \delta C}{Y_{2} C}\right)
$$

Substituting $\delta C$ and $\delta Y_{2}$ by their expressions and taking the ensemble averages, we get

$$
\begin{aligned}
\left\langle\left(Y_{2 F}-\overline{Y_{2 F}}\right)^{2}\right\rangle= & \frac{\overline{Y_{2 F}^{2}}}{N^{2}}\left[\frac{\left\langle\left(\sum_{n=1}^{\infty}\left(\left(\begin{array}{c}
n \\
2
\end{array}\right)-n \bar{C}\right) \varepsilon_{n}\right)^{2}\right\rangle}{\bar{Y}_{2}^{2}}\right. \\
& +\frac{\left\langle\left(\sum_{n=1}^{\infty} n \varepsilon_{n}\right)^{2}\right\rangle}{C^{2}} \\
& \left.-2 \frac{\left\langle\left(\sum_{n=1}^{\infty}\left(\begin{array}{c}
n \\
2
\end{array}\right)-n \bar{C}\right) \varepsilon_{n} \sum_{n=1}^{\infty} n \varepsilon_{n}\right\rangle}{Y_{2} C}\right]
\end{aligned}
$$

Fig. 3 shows the Feynman variance $\overline{Y_{2 F}}$ computed for a simulation. $\overline{Y_{2 F}}$ as well as its variance are computed as many times as the number of time gates using Eq. 12 and 17, respectively.

The analytical expression for $Y_{2 F}(T)$ from the point model theory is

$$
Y_{2 F}(T)=\varepsilon\left(M-\frac{M-1}{\bar{v}}\right)\left(D_{2 s}+(M-1) D_{2}\right)\left(1-\frac{1-e^{-\lambda T}}{\lambda T}\right)
$$

The asymptote of this expression is

$$
R_{2 F}=\lim _{T \rightarrow \infty} Y_{2 F}(T)=\left(M-\frac{M-1}{\bar{v}}\right)\left(D_{2 s}+(M-1) D_{2}\right) .
$$

The green curve in Fig. 3 shows the fit to the Feynman variance $Y_{2 F}(T)$ using Eq. 18. The fit parameters $\lambda$ and $R_{2 F}$ along with their standard deviation are shown in the figure. Once $R_{2 F}$ is known, Eq. 19 can be used to determine the value of the multiplication $\mathrm{M}$ along with its standard deviation.

In summary, if we know the californium source strength, we can determine the efficiency $\varepsilon$ and multiplication M from Eqs. 11 and 19. 


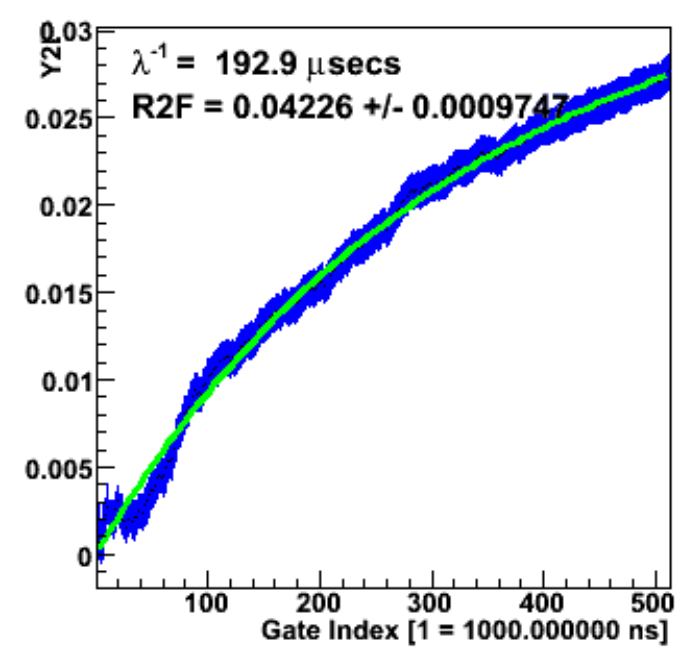

Figure 3: Feynman variance $\overline{Y_{2 F}}(T)$, fit by Eq. 18

\section{Assumptions made for the analysis}

For the analysis, we will assume that we know the source isotope $\left({ }^{252} C f\right)$ and its strength $\left(1.2 \times 10^{6}\right.$ neutrons/second). We will also assume that the source is surrounded by some multiplying medium. We will pick uranium, in which ${ }^{235} U$ multiplies the number of neutrons by inducing fissions. Given that the simulation was performed with no multiplying medium around the object, the analysis should tell us that the system was actually not multiplying, that its multiplication $\mathrm{M}$ was 1 .

\section{Nuclear data}

Equations 11 and 18 contain several parameters that need yet to be discussed. $\bar{v}$ is the average number of neutrons emitted per induced fission, and is equal to 2.52 for ${ }^{235} U . \bar{v}_{s}$ is the average number of neutrons emitted per spontaneous fission. In the case of californium-252, $\bar{v}_{s}=3.77 . D_{2 s}$ is the ratio of the second combinatorial moment of the spontaneous fission neutron number distribution $v_{2 s}$ to $\bar{v}_{s} . D_{2}$ is its counterpart for induced fission. $v_{2 s}=6.02$ for ${ }^{252} C f$, while $v_{2}=2.55$ for ${ }^{235} U$.

\section{Simulations}

Nine simulations of the same californium-252 neutron source in 6" of polyethylene were carried out with different random number generator seeds with MCNPX. Each simulation was 10 minutes long. Helium-3 tubes were placed around the polyethylene to capture the thermal neutrons. Neutrons are captured by ${ }^{3} \mathrm{He}$ via the reaction $n\left({ }^{3} \mathrm{He}, p\right)^{3} \mathrm{H}$. The capture time tags were saved in list-mode in 9 output files. These files were subsequenlty analyzed using the method explained in section 2 and the assumptions in section 4 . 


\section{Overlapping time gates}

The time tagged data was analyzed different ways. Because the analysis for the first 2 cases differs greatly from the analysis for the last 3 cases, we will discuss them separately.

For the first 2 cases, we use the original method of analysing the data, which was originally constrained by the hardware available at the time. The data stream was sliced into $\mathrm{N}$ segments of equal widths $(512 \mu s$ for instance). Counting the number of counts in the first microsecond of each one of these $\mathrm{N}$ segments, one can build a count distribution for a gate width of $1 \mu \mathrm{s}$. An example of such a distribution was shown in Fig. 1. Counting the number of counts in the first two microseconds of each one of these $\mathrm{N}$ segments, one would build a count distribution of gate width $2 \mu \mathrm{s}$, and so on, until we get 512 different count distributions. Of course, because the count distribution for the $2 \mu \mathrm{s}$ time gate used in part the same data as the count distribution for the $1 \mu \mathrm{s}$ time gate, the $2 \mu \mathrm{s}$ count distribution was not independent from the $1 \mu \mathrm{s}$ count distribution, and so on for all the time gates. The moments of the count distributions were then computed and fit with Eqs. 11 and 18. The standard deviation on $R_{2 F}$ was taken to be equal to the standard deviation on the last time gate (the $512^{\text {th }}$ time gate). This choice for the standard deviation was not a choice of the author, but the method conventionally used.

\subsection{2 time gates in arithmetic progression from 1 to $512 \mu \mathrm{s}$}

Using 512 time gates increasing arithmetically from 1 to $512 \mu \mathrm{s}$ in increments of $1 \mu \mathrm{s}$, we get the results in Table 1 for the 9 simulations:

\begin{tabular}{|c||c|c|c|}
\hline simulation & M & M range & $1 / \lambda[\mu s]$ \\
\hline \hline 1 & 0.998267 & {$[0.961865: 1.034644]$} & 192.9 \\
\hline 2 & 1.268506 & {$[1.232080: 1.304907]$} & 296 \\
\hline 3 & 0.731006 & {$[0.694775: 0.767261]$} & 163.1 \\
\hline 4 & 1.000122 & {$[0.963721: 1.036523]$} & 193 \\
\hline 5 & 1.005347 & {$[0.968994: 1.041699]$} & 215.5 \\
\hline 6 & 1.419678 & {$[1.383496: 1.455859]$} & 338.7 \\
\hline 7 & 1.297363 & {$[1.261328: 1.333374]$} & 279.5 \\
\hline 8 & 0.853955 & {$[0.817627: 0.890308]$} & 176.3 \\
\hline 9 & 0.945630 & {$[0.909131: 0.982129]$} & 191.6 \\
\hline
\end{tabular}

Table 1: Multiplication for 9 different simulations using 512 time gates in arithmetic progression from $1 \mu s$ to $512 \mu \mathrm{s}$.

The $3^{\text {rd }}$ column gives the range of multiplication M obtained using $R_{2 F}+/-\sigma$. One should note that the values of the multiplication $\mathrm{M}$ were in no way constrained to be greater than 1 , as they should be physically. $\mathrm{M}$ was computed mathematically by solving the 2 equations above.

\subsection{2 time gates in arithmetic progression from 4 to $2000 \mu \mathrm{s}$}

We observe in Table 1 that the time constant $\lambda^{-1}$ is of the order of $200 \mu s$. To get a more accurate value of $\mathrm{M}$, it would be better to compute the Feynman variance on a time gate range that goes at least up to 10 times $\lambda^{-1}$. Indeed, while $512 \mu \mathrm{s}$ is not high enough to get a good fit for $\overline{Y_{2 F}}(T)$ when the $\lambda^{-1}$ time scale is of the order of $200 \mu s, 2000 \mu s$ should be sufficient. Given that each $2000 \mu s$ segment is 4 times longer than 
the previous $512 \mu s$, the number of samples in the new count distribution $b_{n}$ will be decreased by a factor 4 . Using 512 time gates increasing arithmetically from 4 to $2000 \mu s$ in increments of $4 \mu s$, we get the results in Table 2:

\begin{tabular}{|c||c|c|c|}
\hline simulation & M & M range & $1 / \lambda[\mu s]$ \\
\hline \hline 1 & 1.11333 & {$[1.039648: 1.187012]$} & 231.9 \\
\hline 2 & 0.996387 & {$[0.922697: 1.070190]$} & 180.7 \\
\hline 3 & 0.925586 & {$[0.852686: 0.998462]$} & 206.9 \\
\hline 4 & 1.11333 & {$[1.039648: 1.187012]$} & 232.0 \\
\hline 5 & 1.076636 & {$[1.003296: 1.150000]$} & 229.4 \\
\hline 6 & 1.057666 & {$[0.984082: 1.131201]$} & 253.7 \\
\hline 7 & 0.955396 & {$[0.882080: 1.028711]$} & 209.3 \\
\hline 8 & 0.846191 & {$[0.773462: 0.918945]$} & 139.0 \\
\hline 9 & 1.018555 & {$[0.945703: 1.091382]$} & 205.5 \\
\hline
\end{tabular}

Table 2: Multiplication for 9 different simulations using 512 time gates in arithmetic progression from $4 \mu \mathrm{s}$ to $2000 \mu s$.

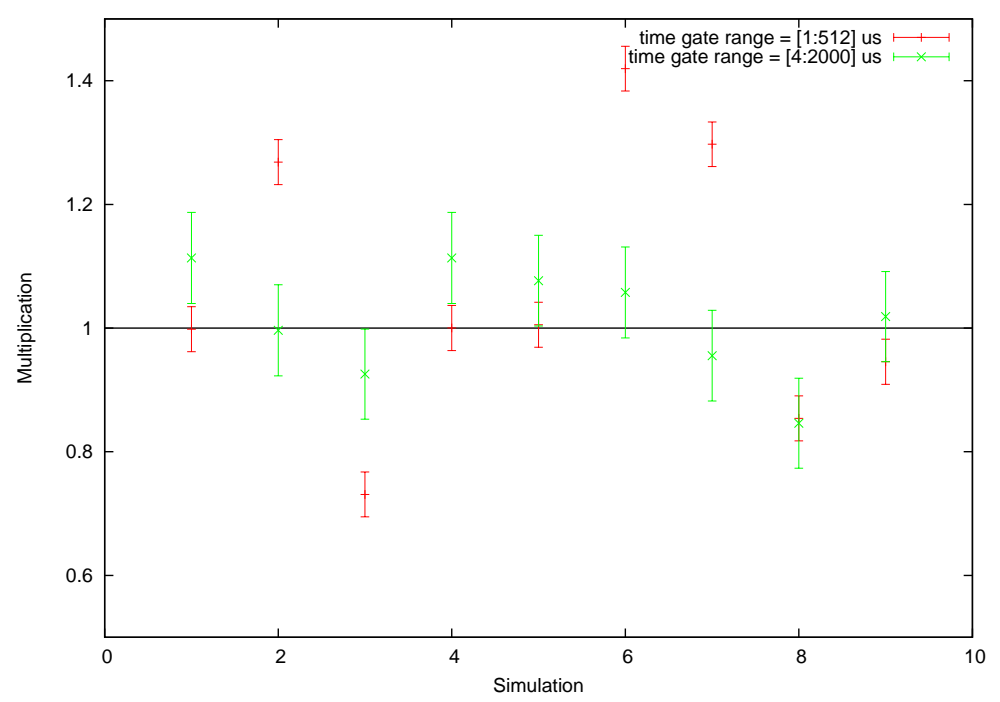

Figure 4: Multiplication ranges for the two different gate width ranges

Several observations need to be made on Fig. 4, which shows the range of the multiplication M obtained for the two choices of time gate ranges. The larger time gate range results in multiplications much closer to the true value of 1 than the shorter time gate range. Also, the spread of the multiplication $\mathrm{M}$ is much narrower for the longer time gate range. As a conclusion, a good choice of time gate range based on the value of $\lambda^{-1}$ is important. One should note however that the number of multiplication intervals including the right answer $\mathrm{M}=1$ is 3 and 4 for the short and larger time gate ranges, respectively, which is less than the expected value of $0.68 * 9=6$ for a 1 sigma confidence interval. The standard deviation on the multiplication seems to be too small, which is not surprising, given that the standard deviation on $R_{2 F}$ was taken to be equal to the 
standard deviation on the last time gate of $\overline{Y_{2 F}}(T)$, which is incorrect. The rest of this report revisits the way the standard deviation on $R_{2 F}$ is estimated.

\section{Non-overlapping time gates}

One of the deficiencies of the method used above was that the same data was used over and over to compute the count distributions for all the time gates. Therefore, all the points along the $\overline{Y_{2 F}}(T)$ were correlated and it was difficult to the standard deviation on the fit parameters $\mathrm{M}$ and $\varepsilon$ correctly.

Instead of reusing the same data over and over for the different time gates, we now propose to compute each count distribution from its own data segment, and this data segment will not be used by any other count distribution. This is how the algorithm is implemented in practice. The first task is to choose a set of time gate widths for which we wish to compute count distributions. In the 3 examples below, we picked 57, 60 and 63 times gates widths ranging from $1 \mathrm{~ns}$ to $524 \mu s, 1048 \mu \mathrm{s}$, and $2096 \mu \mathrm{s}$, respectively. As the data arrives, we first pick randomly which time gate to open. Once a time gate of width $\mathrm{T}$ has been chosen, we count how many events arrive within the time interval $\mathrm{T}$, and update the corresponding count distribution. We then pick another time gate randomly for the next data segment. Thus, we get a number of count distributions $b_{n}$ that will have been built from different sets of the entire data stream. $\overline{Y_{2 F}}$ can then be computed for each of these count distributions $b_{n}$. Because each of these $\overline{Y_{2 F}}$ values will be independent of each other, we can use Minuit [2] to fit the data with Eq. 18. Minuit will also produce the standard deviations around the fit parameters $M$ and $\lambda^{-1}$. This could not be done using the other technique and independence between the data points is required for the standard deviation to be computed correctly.

N.B.: After doing this work, I realized that it would be more adequate for the smallest time gate to be $1 \mu \mathrm{s}$ instead of $1 \mathrm{~ns}$. Anything below $1 \mu \mathrm{s}$ (let alone $1 \mathrm{ns)} \mathrm{makes} \mathrm{no} \mathrm{sense} \mathrm{for} \mathrm{thermal} \mathrm{neutrons.}$ Also, using $1 \mathrm{~ns}$ in general breaks the assumption in the point-model theory that the fission chain neutrons are all emitted instantaneously compared to the diffusion time scale of the neutrons through the surrounding material to the detector. While this is not a problem with ${ }^{252} \mathbf{C f}$, because there are no induced fissions and we can assume that the spontaneous fission neutrons are emitted instantaneously compared to the $1 \mathrm{~ns}$ "diffusion time scale", this would lead to problems for multiplying systems.

\subsection{7 non-overlapping time gates in geometric progression from $1 \mathrm{~ns}$ to $524 \mu \mathrm{s}$}

Using 57 non overlapping time gates increasing in size using a geometric progression from $1 \mathrm{~ns}$ to $524 \mu \mathrm{s}$, we get $\overline{Y_{2 F}}$ shown in Fig. 5 for the first (out of nine) simulation. The data is fit by Eq. 18, and the standard deviations on $\lambda^{-1}$ and $R_{2 F}$ shown in the figure are computed by Minuit.

The results for the 9 simulations are summarized in Table 3:

We observe in Table 3 that the standard deviation on $\lambda^{-1}$ is large. This is a consequence of the largest count distribution time interval (524 $\mu s$ ) being too small compared to the value of $\lambda^{-1}$. The next 2 sections will use the same method of non-overlapping time intervals but for larger time intervals.

\subsection{0 non-overlapping time gates in geometric progression from $1 \mathrm{~ns}$ to $1048 \mu \mathrm{s}$}

Using 60 non overlapping time gates increasing in size using a geometric progression from 1 ns to $1048 \mu s$, we get the results in Table 4. 


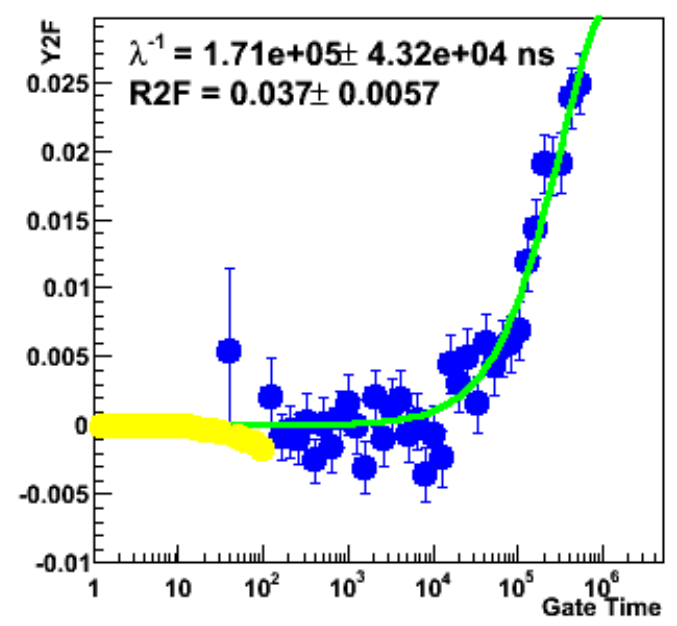

Figure 5: Feynman variance $\overline{Y_{2 F}}(T)$, fit by Eq. 18

\begin{tabular}{|c||c|c|c|}
\hline Simulation & M & M range & $1 / \lambda[\mu s]$ \\
\hline \hline 1 & 0.80752 & {$[0.596191: 1.018848]$} & $171 \pm 43$ \\
\hline 2 & 1.499243 & {$[0.829102: 2.169385]$} & $325 \pm 130$ \\
\hline 3 & 1.577588 & {$[0.807837: 2.347363]$} & $344 \pm 147$ \\
\hline 4 & 0.807153 & {$[0.595874: 1.018457]$} & $171 \pm 43$ \\
\hline 5 & 1.185229 & {$[0.790601: 1.579883]$} & $246 \pm 78$ \\
\hline 6 & 0.995776 & {$[0.661304: 1.330225]$} & $211 \pm 68$ \\
\hline 7 & 1.110181 & {$[0.656763: 1.563574]$} & $267 \pm 97$ \\
\hline 8 & 0.74939 & {$[0.546289: 0.95249]$} & $162 \pm 42$ \\
\hline 9 & 2.592871 & {$[0.583569: 4.602246]$} & $525 \pm 302$ \\
\hline
\end{tabular}

Table 3: Multiplication for 9 different simulations using 57 non-overlapping time gates in geometric progression from $1 \mathrm{~ns}$ to $524 \mu \mathrm{s}$.

Table 4 shows values of $\lambda^{-1}$ that fluctuate much less than in the previous case. The standard deviations on $\lambda^{-1}$ are much smaller as well. In the next section, we will increase the largest count distribution time gate to be $2096 \mu s$, which is about 10 times the value of $\lambda^{-1}$.

\subsection{3 non-overlapping time gates in geometric progression from 1 ns to $2096 \mu \mathrm{s}$}

Using 63 non-overlapping time gates increasing in width geometrically from 1 ns to $2096 \mu \mathrm{s}$, we get the results in Table 5 .

The values of $\lambda^{-1}$ shown in Table 5 are well behaved, all the values of $\lambda^{-1}$ are within a small interval. The multiplication ranges from the last 3 tables are plotted in Fig. 6.

We observe in Fig. 6 that 9 out of 9 multiplication intervals for the 57 gate case encompass the the right value $M=1,5$ out of 9 for the 60 gate case, and 8 out of 9 for the 63 gate case. These 3 ratios are close to what we would expect for a 1 sigma confidence interval. This result is much better than what we observed in Fig. 4 for the overlapping time gates. 


\begin{tabular}{|c||c|c|c|}
\hline Simulation & M & M range & $1 / \lambda[\mu s]$ \\
\hline \hline 1 & 1.290674 & {$[1.036377: 1.544971]$} & $302 \pm 72$ \\
\hline 2 & 1.285400 & {$[1.039282: 1.531494]$} & $304 \pm 68$ \\
\hline 3 & 1.025391 & {$[0.846265: 1.204492]$} & $231 \pm 49$ \\
\hline 4 & 1.290674 & {$[1.036377: 1.544971]$} & $302 \pm 72$ \\
\hline 5 & 1.138916 & {$[0.959668: 1.318213]$} & $227 \pm 47$ \\
\hline 6 & 1.026904 & {$[0.840137: 1.213623]$} & $237 \pm 53$ \\
\hline 7 & 0.915308 & {$[0.762134: 1.068506]$} & $192 \pm 42$ \\
\hline 8 & 1.328662 & {$[1.055615: 1.601758]$} & $319 \pm 78$ \\
\hline 9 & 1.137842 & {$[0.962427: 1.313281]$} & $228 \pm 46$ \\
\hline
\end{tabular}

Table 4: Multiplication for 9 different simulations using 60 non-overlapping time gates in geometric progression from $1 \mathrm{~ns}$ to $1048 \mu \mathrm{s}$.

\begin{tabular}{|c||c|c|c|}
\hline Simulation & M & M range & $1 / \lambda[\mu s]$ \\
\hline \hline 1 & 1.011475 & {$[0.881812: 1.141113]$} & $213 \pm 44$ \\
\hline 2 & 1.069775 & {$[0.933667: 1.205908]$} & $239 \pm 48$ \\
\hline 3 & 1.037328 & {$[0.877368: 1.195288]$} & $254 \pm 61$ \\
\hline 4 & 1.011841 & {$[0.882178: 1.141504]$} & $213 \pm 44$ \\
\hline 5 & 1.091406 & {$[0.950903: 1.231934]$} & $244 \pm 50$ \\
\hline 6 & 0.951025 & {$[0.822119: 1.079932]$} & $203 \pm 45$ \\
\hline 7 & 0.893359 & {$[0.763599: 1.02312]$} & $199 \pm 46$ \\
\hline 8 & 1.022412 & {$[0.87981: 1.16499]$} & $236 \pm 52$ \\
\hline 9 & 1.152954 & {$[1.021411: 1.284473]$} & $229 \pm 43$ \\
\hline
\end{tabular}

Table 5: Multiplication for 9 different simulations using 63 non-overlapping time gates in geometric progression from $1 \mathrm{~ns}$ to $2096 \mu \mathrm{s}$.

Another interesting point is that even though the shortest gate width interval 1 ns to $524 \mu \mathrm{s}$ has four times the number of samples as the longest gate width interval $1 \mathrm{~ns}$ to $2096 \mu \mathrm{s}$, the standard deviation on the multiplication $\mathrm{M}$ is much larger. This reiterates the fact that the largest time gate width has to be much larger than the characteristic $\lambda^{-1}$ of the problem.

\section{Conclusion}

The results of this study show that analyzing the data stream using non-overlapping time gates is an improvement over the current analysis using overlapping time gates. The new method enables us to compute the standard deviation on the multiplication parameter $\mathrm{M}$ accurately. Also, this standard deviation on $\mathrm{M}$ decreases as the largest time gate is about 10 times the characteristic time constant $\lambda^{-1}$ of the system.

It was also shown that the analysis accurately predicts a multiplication of 1 for ${ }^{252} C f$ in a non-multiplying configuration. 


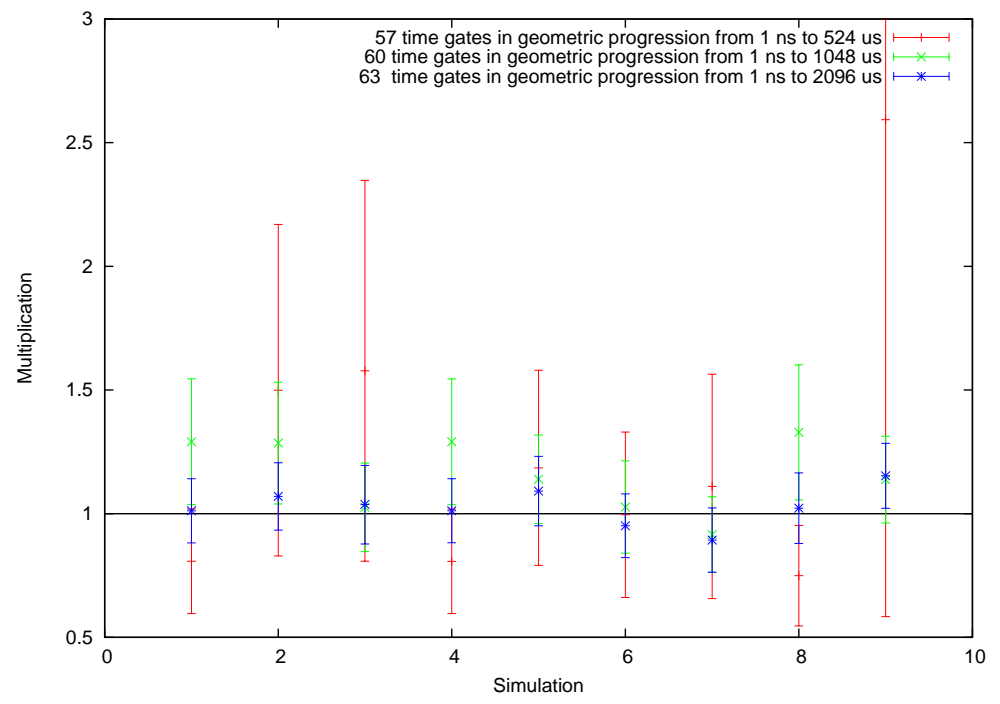

Figure 6: Multiplication ranges for the three different gate width ranges: 57 time gates from 1 ns to 524 $\mu s$ in geometric progression (red), 60 time gates from $1 \mathrm{~ns}$ to $1048 \mu s$ in geometric progression (green), 63 time gates from $1 \mathrm{~ns}$ to $2096 \mu \mathrm{s}$ in geometric progression (blue). 


\section{References}

[1] M.O. Deighton, "Theoretical Comparion of the Enhanced Variance and Twin-Gate Methods for Monitoring Plutonium in Waste," Nuclear Instruments and Methods, 165, 589-605 (1979).

[2] F. James and M. Winkler, "MINUIT User's Guide," http://seal.web.cern.ch/seal/documents/minuit/mnusers. 